\title{
Geometric accuracy of an acrylonitrile butadiene styrene canine tibia model fabricated using fused deposition modelling and the effects of hydrogen peroxide gas plasma sterilisation
}

Chi-Pin Hsu', Chen-Si Lin², Chun-Hao Fan ${ }^{3}$, Nai-Yuan Chiang ${ }^{4}$, Ching-Wen Tsai', Chun-Ming Chang ${ }^{4}$ and I-Li Liü $2,3^{*}$ (D)

\begin{abstract}
Background: Three-dimensional (3D) printing techniques have been used to produce anatomical models and surgical guiding instruments in orthopaedic surgery. The geometric accuracy of the 3D printed replica may affect surgical planning. This study assessed the geometric accuracy of an acrylonitrile butadiene styrene (ABS) canine tibia model printed using fused deposition modelling (FDM) and evaluated its morphological change after hydrogen peroxide $\left(\mathrm{H}_{2} \mathrm{O}_{2}\right)$ gas plasma sterilisation. The tibias of six canine cadavers underwent computed tomography for 3D reconstruction. Tibia models were fabricated from ABS on a 3D printer through FDM. Reverseengineering technology was used to compare morphological errors (root mean square; RMS) between the 3D-FDM models and virtual models segmented from original tibia images (3D-CT) and between the models sterilised with $\mathrm{H}_{2} \mathrm{O}_{2}$ gas plasma (3D-GAS) and 3D-FDM models on tibia surface and in cross-sections at: $5,15,25,50,75,85$, and 95\% of the tibia length.

Results: The RMS mean \pm standard deviation and average positive and negative deviation values for all specimens

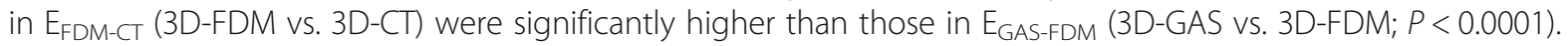
Mean RMS values for $\mathrm{E}_{\mathrm{FDM}-\mathrm{CT}}$ at $5 \%$ bone length (proximal tibia) were significantly higher than those at the other six cross-sections $(P<0.0001)$. Mean RMS differences for $E_{G A S-F D M}$ at all seven cross-sections were nonsignificant.

Conclusions: The tibia models fabricated on an FDM printer had high geometric accuracy with a low RMS value. The surface deviation in $\mathrm{E}_{\mathrm{FDM}-\mathrm{CT}}$ indicated that larger errors occurred during manufacturing than during sterilisation. Therefore, the model may be used for surgical rehearsal and further clinically relevant applications in bone surgery.
\end{abstract}

Keywords: Fused deposition modelling, Long bone model, Sterilisation, Surgical guide, Three-dimensional printing

\footnotetext{
* Correspondence: liuili@ntu.edu.tw

2Department and Graduate Institute of Veterinary Medicine, School of Veterinary Medicine, National Taiwan University, Taipei, Taiwan

${ }^{3}$ Institute of Veterinary Clinical Science, School of Veterinary Medicine, National Taiwan University, Taipei, Taiwan

Full list of author information is available at the end of the article
}

C The Author(s). 2020 Open Access This article is licensed under a Creative Commons Attribution 4.0 International License, which permits use, sharing, adaptation, distribution and reproduction in any medium or format, as long as you give appropriate credit to the original author(s) and the source, provide a link to the Creative Commons licence, and indicate if changes were made. The images or other third party material in this article are included in the article's Creative Commons. licence, unless indicated otherwise in a credit line to the material. If material is not included in the article's Creative Commons licence and your intended use is not permitted by statutory regulation or exceeds the permitted use, you will need to obtain permission directly from the copyright holder. To view a copy of this licence, visit http://creativecommons.org/licenses/by/4.0/ The Creative Commons Public Domain Dedication waiver (http://creativecommons.org/publicdomain/zero/1.0/) applies to the data made available in this article, unless otherwise stated in a credit line to the data. 


\section{Background}

Three-dimensional (3D) printing techniques have been used extensively for anatomical models, presurgical planning, surgical guiding instruments, custom-made implants, and client communication in human medicine, particularly in orthopaedic, facial reconstructive, spinal, and dental surgery [1-3]. Presurgical planning using 3Dprinting anatomical models has been adopted in veterinary medicine [4-6] and related research [7, 8], particularly in orthopaedic surgery, thus improving surgeon confidence and reducing surgical time, the occurrence of surgical complications, and surgeons' exposure to radiation $[4,6,8]$. These studies have indicated that 3Dprinted models are beneficial when used for managing complex cases and when used by inexperienced surgeons $[4,6,9]$. Additionally, 3D-printed bone models are suitable for presurgical rehearsals of plate contouring, osteotomy, and the design of surgical guides $[4,6,7,9]$. However, in veterinary orthopaedics, only three studies have evaluated the linear deviation of 3D-printed longbone models [10-12]. Moreover, the geometric accuracy of 3D-printed long bones, which is crucial for bone-plate contouring and the design of patient-specific surgical guides, has not been evaluated.

The majority of commercially available desktop printers for rapid prototyping are based on fused deposition modelling (FDM). These types of desktop printers comprise $46 \%$ of all 3D printing devices in use [13]. They are easy to use and suitable for offices, providing prints of similar accuracy to industrial printers $[14,15]$. Consumer-grade FDM printers have been used in some studies to produce inexpensive maxillofacial and long-bone models in an office setting $[11,12]$. Other studies have demonstrated that 3D-printing of patient-specific guides is a safe, promising, and affordable method that achieves satisfactory clinical outcomes $[16,17]$. Even though the plastic filament used in $3 \mathrm{D}$ printing has a low melting point, given the printed models intraoperative usage, sterilisation of plastic models using an autoclave has been suggested [3, 12]. Studies, however, have demonstrated deformation of plastic models after steaming [18-20] or alteration to their mechanical strength after gas plasma sterilisation [21]. This evidence suggests such plastic filaments are problematic since they are sensitive to conventional thermal steam sterilisation. However, little is known regarding the accuracy and morphological changes of these plastic bone models after sterilisation. Because this is a new field, further research is warranted [1].

Designing patient-specific surgical tools for canine tibial plateau levelling osteotomy (TPLO), including 3Dprinted tibias and bone-cutting instruments, is our field of interest in veterinary medicine. Tibial bone-surface curvature is critical for plate implantation and design of bone-cutting instruments. To the best of our knowledge, no data are currently available regarding the effects of low-temperature sterilisation on the surface accuracy of 3D-printed ABS canine tibias manufactured using FDM. Therefore, an accurate 3D plastic model is crucial for preoperative planning and intraoperative applications, and the extent of plastic model deformation after sterilisation needs evaluation. We focused on hydrogen peroxide $\left(\mathrm{H}_{2} \mathrm{O}_{2}\right)$ low-temperature sterilisation, which is suitable for surgical instruments that are sensitive to heat and moisture [22].

The objectives of this study were (1) to compare the geometric accuracy of an ABS tibia model 3D printed using FDM with that of a 3D tibia model derived using computed tomography $(\mathrm{CT})$ and (2) to evaluate possible geometric change of the 3D ABS tibia model after sterilisation with $\mathrm{H}_{2} \mathrm{O}_{2}$ gas plasma.

\section{Results}

\section{Geometric deviation of the whole-tibia model}

Table 1 and Fig. 1 provide superimposition results of the RMS values for 12 tibias in the two comparison groups: $\mathrm{E}_{\mathrm{FDM}-\mathrm{CT}}$ (3D-FDM vs. 3D-CT) and $\mathrm{E}_{\mathrm{GAS}-\mathrm{FDM}}$ (3D-GAS vs. 3D-FDM). The RMS mean \pm standard deviation and average positive and negative deviation values for all specimens in $\mathrm{E}_{\mathrm{FDM}-\mathrm{CT}}(0.1214 \pm 0.0185 \mathrm{~mm}, 0.0973 \pm$ $0.0182 \mathrm{~mm}$, and $-0.0737 \pm 0.0170 \mathrm{~mm}$, respectively) were significantly higher than those in $\mathrm{E}_{\mathrm{GAS}-\mathrm{FDM}}(0.0431 \pm$ $0.0248 \mathrm{~mm}, 0.0234 \pm 0.0162 \mathrm{~mm}$, and $-0.0258 \pm 0.0196$ $\mathrm{mm}, P<0.0001$, respectively; Table 2 ). These results indicate that the difference that occurred during $3 \mathrm{D}$ printing was significantly larger than the deviation that occurred after $\mathrm{H}_{2} \mathrm{O}_{2}$ plasma sterilisation. Therefore, the

Table $13 \mathrm{D}$ comparison of 3D-CT, 3D-FDM, and 3D-GAS of the tibia models

\begin{tabular}{lll}
\hline Case & $\begin{array}{l}E_{F D M-C T} \text { (3D-FDM vs. 3D-CT) } \\
\text { RMS value (mm) }\end{array}$ & $\begin{array}{l}E_{\text {GAS-FDM (3D-GAS vs. 3D-FDM) }} \\
\text { RMS value (mm) }\end{array}$ \\
\hline No.1 & 0.1300 & 0.0373 \\
No.2 & 0.1564 & 0.0568 \\
No.3 & 0.1463 & 0.0284 \\
No.4 & 0.1151 & 0.1123 \\
No.5 & 0.1292 & 0.0301 \\
No.6 & 0.1323 & 0.0320 \\
No.7 & 0.0910 & 0.0223 \\
No.8 & 0.1074 & 0.0258 \\
No.9 & 0.1024 & 0.0614 \\
No.10 & 0.1178 & 0.0402 \\
No.11 & 0.1099 & 0.0398 \\
No.12 & 0.1194 & 0.0311 \\
\hline
\end{tabular}

3D-FDM Images obtained from reverse-scanned 3D-printed tibia models before sterilisation, $3 D-C T$ Original tibia images obtained from $C T, 3 D-G A S$ Images obtained from reverse-scanned 3D-printed tibia models after sterilisation, RMS Root mean square 


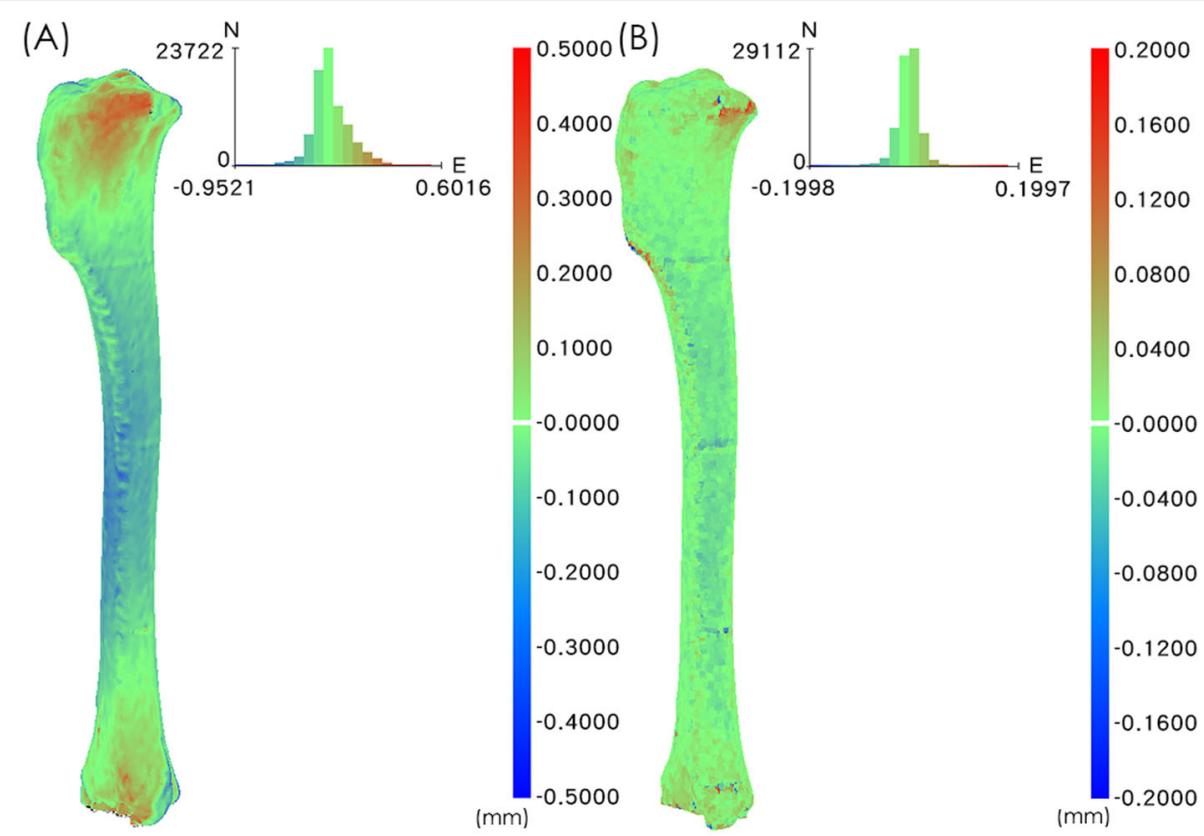

Fig. 1 The colour difference map represents the difference between the tibial bone models. a Superposition of 3D-FDM and 3D-CT tibia models in case No.3. The maximum and minimum values of the colour difference map were +0.5000 and $-0.5000 \mathrm{~mm}$. $\mathbf{b}$ Superposition of 3D-GAS and 3D-FDM tibia models in case No.3. The maximum and minimum values of the colour difference map were $+0.2000 \mathrm{and}-0.2000 \mathrm{~mm}$

Table 2 Mean \pm standard deviation values for comparisons of measurements obtained from 3D-CT, 3D-FDM, and 3D-GAS images of the tibial bone surfaces $(n=12)$ of six canine cadavers

\begin{tabular}{|c|c|c|}
\hline Variable & $\mathrm{E}_{\mathrm{FDM}-\mathrm{CT}}(3 \mathrm{D}-\mathrm{FDM}$ vs. 3D-CT) & $\mathrm{E}_{\text {GAS-FDM }}$ (3D-GAS vs. 3D-FDM) \\
\hline \multicolumn{3}{|l|}{ Surface deviation } \\
\hline Root mean square $(\mathrm{mm})$ & $0.1214 \pm 0.0185^{\mathrm{a}}$ & $0.0431 \pm 0.0248$ \\
\hline Average deviation positive (mm) & $0.0973 \pm 0.0182^{\mathrm{a}}$ & $0.0234 \pm 0.0162$ \\
\hline Average deviation negative (mm) & $-0.0737 \pm 0.0170^{a}$ & $-0.0258 \pm 0.0196$ \\
\hline \multicolumn{3}{|c|}{ Curvature difference of cross-sectional measurements of bone surface at: } \\
\hline (a) $5 \%$ length of the tibia $(\mathrm{mm})$ & $0.3025 \pm 0.2159^{b}$ & $0.0491 \pm 0.0428$ \\
\hline (b) $15 \%$ length of the tibia (mm) & $0.0863 \pm 0.0227$ & $0.0273 \pm 0.0180$ \\
\hline (c) $25 \%$ length of the tibia $(\mathrm{mm})$ & $0.0645 \pm 0.0186$ & $0.0247 \pm 0.0137$ \\
\hline (d) $50 \%$ length of the tibia (mm) & $0.1313 \pm 0.0449$ & $0.0376 \pm 0.0404$ \\
\hline (e) $75 \%$ length of the tibia $(\mathrm{mm})$ & $0.1307 \pm 0.0385$ & $0.0263 \pm 0.0194$ \\
\hline (f) $85 \%$ length of the tibia $(\mathrm{mm})$ & $0.1020 \pm 0.0353$ & $0.0239 \pm 0.0160$ \\
\hline (g) $95 \%$ length of the tibia (mm) & $0.1288 \pm 0.0674$ & $0.0351 \pm 0.0346$ \\
\hline
\end{tabular}


change due to $\mathrm{H}_{2} \mathrm{O}_{2}$ plasma sterilisation was sufficiently small to disregard.

\section{Cross-sectional profile deviation of the tibia model}

Figure 2 is a graph of the $\mathrm{E}_{\mathrm{FDM}-\mathrm{CT}}$ and $\mathrm{E}_{\mathrm{GAS}-\mathrm{FDM}}$ crosssectional profiles at $5 \%$ of the total tibia length. Additionally, the mean RMS values of $E_{\mathrm{FDM}-\mathrm{CT}}$ at $5 \%$ of the total bone length $(0.3025 \pm 0.2159 \mathrm{~mm})$ were significantly higher than those at the other six cross-sections (15\%: $0.0863 \pm 0.0227 \mathrm{~mm} ; \quad 25 \%$ : $0.0645 \pm 0.0186 \mathrm{~mm}$; 50\%: $0.1313 \pm 0 \quad .0449 \mathrm{~mm} ; \quad 75 \%$ : $0.1307 \pm 0.0385 \mathrm{~mm}$; 85\%: $0.1020 \pm 0.0353 \mathrm{~mm}$; and 95\%: $0.1288 \pm 0.0674 \mathrm{~mm}$; $P<0.0001)$. The differences in mean RMS values of $\mathrm{E}_{\mathrm{GAS}-\mathrm{FDM}}$ at all seven cross-sections were not significant (Table 2 and Fig. 3). Therefore, no regional deviation was observed in the tibia model after $\mathrm{H}_{2} \mathrm{O}_{2}$ plasma sterilisation.

\section{Discussion}

Some surgical studies have reported the application of 3D plastic models as surgical guides for intraoperative use $[5,6,9,17]$. Moreover, the geometric accuracy of surgical guides is critical during surgery. Our tibia model had high bone-surface accuracy, which is beneficial for bone-plate contouring and presurgical rehearsal.
Additionally, the surface curvature of the ABS 3Dprinted tibia model was preserved without significant changes after gas plasma sterilisation, indicating possible clinical relevance of our model as a guide during surgery.

Our tibia model fabricated using an FDM printer and measured using a 3D point cloud had high geometric accuracy with a lower mean RMS value $\left(\mathrm{E}_{\mathrm{FDM}-\mathrm{CT}}\right.$ : 0.1214 $\mathrm{mm}$ ) than those of previous studies performing linear RMS measurements on the basis of two-dimensional CT images obtained from 3D plastic long-bone models. These studies have reported RMS values of $0.40 \mathrm{~mm}$ [11] and $<1 \mathrm{~mm}$ [12]. Another study reported that a human mandible model had an absolute mean surface deviation of $0.159 \mathrm{~mm}$ [14], which is consistent with our observations. Given all this, it is evident that bone size can influence model accuracy. Studies have indicated dimensional errors increasing when bone model size increased to $260 \mathrm{~mm}$ in length [11]. They have also shown that an optimal bone model size of $120 \mathrm{~mm}$ in length has better linear accuracy $[11,12]$. The tibial models in the present study are $150-180 \mathrm{~mm}$ in length and have low RMS values. Furthermore, bone surface curvature can also influence model accuracy. Two studies revealed that the largest deviation errors occurred in bone

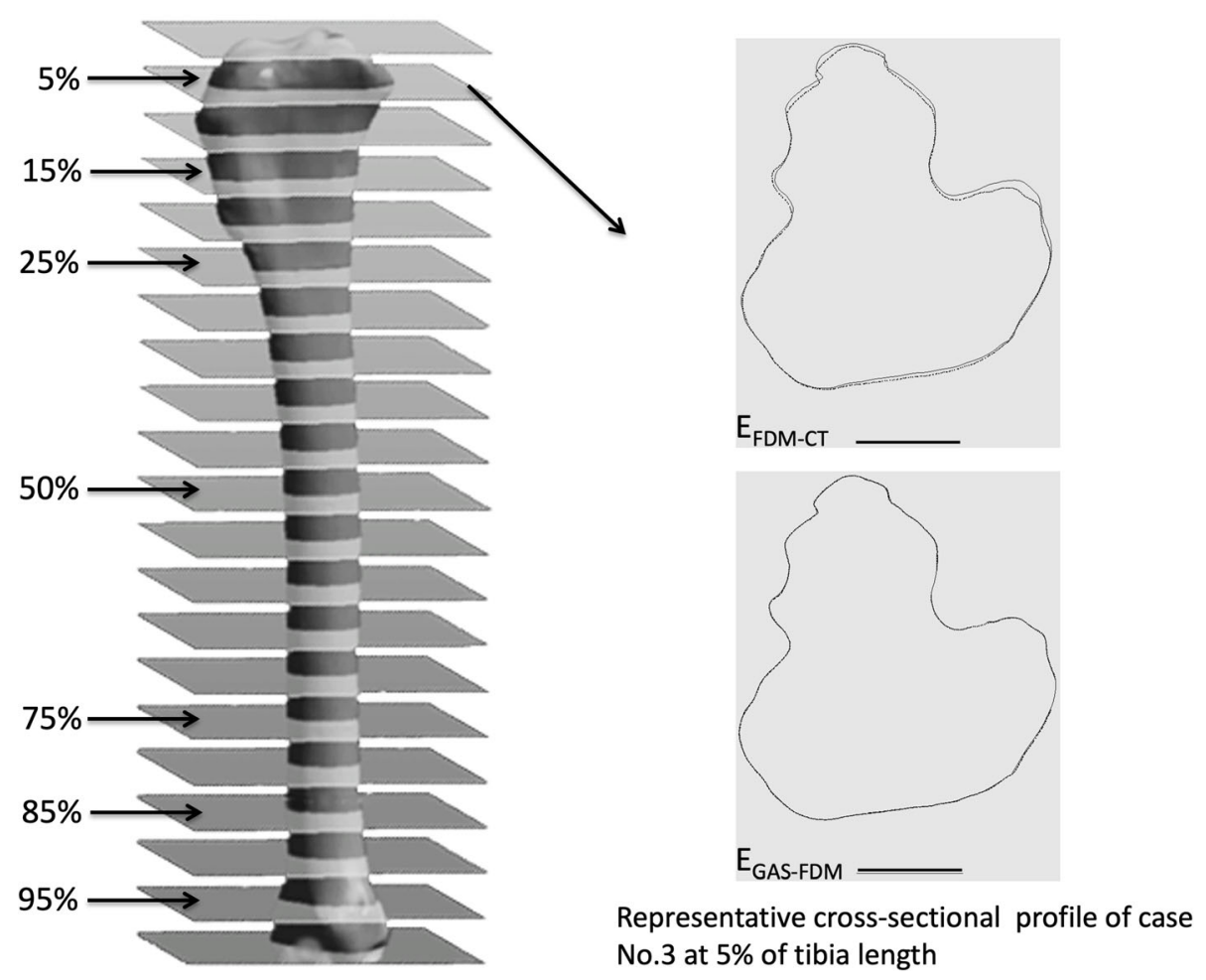

Fig. 2 Three-dimensional tibia model and cross-sectional measurements. Total tibia length was measured between articular surfaces. The crosssectional planes at: 5, 15, 25, 50, 75, 85, and 95\% of the total tibia length were analysed (left). The map represents the difference in cross-sectional profiles of case No.3 in $\mathrm{E}_{\mathrm{FDM}-\mathrm{CT}}$ and $\mathrm{E}_{\mathrm{GAS}-\mathrm{FDM}}$ at $5 \%$ of the tibia length (right). Large deviations occurred in $\mathrm{E}_{\mathrm{FDM}-\mathrm{CT}}$ but not in $\mathrm{E}_{\mathrm{GAS}-\mathrm{FDM}}$. The dotted line denotes the test data, and the solid line denotes the reference data. Scale bar: $10 \mathrm{~mm}$ 


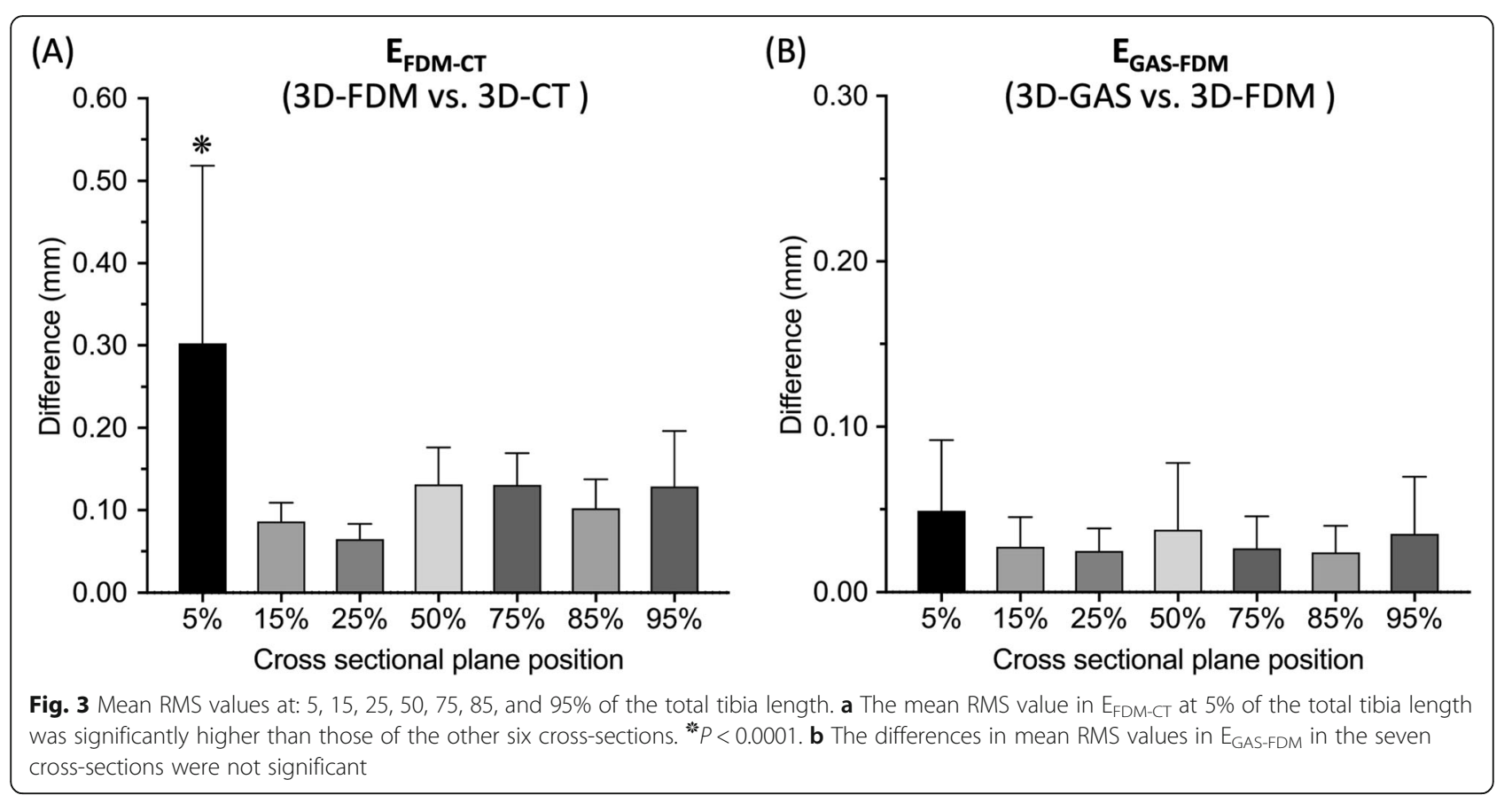

epiphyses with high levels of curvature [23, 24]. This was observed in our $\mathrm{E}_{\mathrm{FDM}-\mathrm{CT}}$ group. The curvature difference of the cross-section at the proximal tibia (5\%) was significantly larger than that of the other cross-sections. Similarly, curvature variations at $15 \%(0.0863 \mathrm{~mm})$ and $25 \%(0.0645 \mathrm{~mm})$ of the total tibia length in $\mathrm{E}_{\mathrm{FDM}-\mathrm{CT}}$ were smaller than those in the other sections (Table 2). These variations may have occurred because tibia geometry at the proximal-medial site was relatively flat. Additionally, the small RMS error may be related to the 3Dprinting build orientation with a $90^{\circ}$ build angle and thick support structure $[25,26]$. These results indicate that the data acquisition method, bone size and geometry, manufacturing process, and material could all have influenced the accuracy of the final model.

Steam heat, dry heat, $\mathrm{H}_{2} \mathrm{O}_{2}$ gas plasma, and ethylene oxide gas sterilisation are clinically used to sterilise plastic surgical models [1,5]. Geometric deviation of sterilised FDM products made of ABS was mentioned in only one study [20]. Uniform rectangular samples sterilised in an autoclave that used steam heat deformed significantly $(>10 \mathrm{~mm})$, and deformations could be observed by the naked eye. However, the average geometric deviation of samples after $\mathrm{H}_{2} \mathrm{O}_{2}$ gas plasma sterilisation was $0.036 \mathrm{~mm}$ [20], which is similar to our observation in $\mathrm{E}_{\mathrm{GAS}-\mathrm{FDM}}(0.043 \mathrm{~mm})$. Our $\mathrm{E}_{\mathrm{GAS}-\mathrm{FDM}}$ results indicate acceptable mean surface deviations with no significant differences between any cross-sections, but plasma sterilisation may have a greater effect on geometric accuracy in more complex anatomical models [20].
The geometric accuracy of 3D-printed models designed for surgical use should be considered because overall errors are influenced by model geometry, printing materials, manufacturing processes, and sterilisation processes [1]. In our ABS canine tibia model, the surface deviation in $\mathrm{E}_{\mathrm{FDM}-\mathrm{CT}}$ indicated that errors occurred during the manufacturing process. These errors were significantly larger than the $\mathrm{E}_{\mathrm{GAS}-\mathrm{FDM}}$ deviations in geometric change after plasma sterilisation. These results indicate that our ABS canine tibia model was fabricated with high accuracy [14] and successfully sterilised using $\mathrm{H}_{2} \mathrm{O}_{2}$ plasma without significant effects on its surface geometry. However, when generating the 3D-CT model from the CT image data, some aggregate sources of errors, such as: CT scan resolution, segmentation methods, and regions of long bone may influence the accuracy of the final 3D virtual bone model. Two studies found different segmentation methods generated results with mean errors of $0.18-0.24 \mathrm{~mm}$ [27] and $1.07 \mathrm{~mm}$ [28], which are higher than the mean errors of $E_{\mathrm{FDM}-\mathrm{CT}}$ $(0.1214 \mathrm{~mm})$ presented here. There was a variety of optimal threshold values for different regions of long bone [27]. Even using advanced methods to process and generate 3D models from $\mathrm{CT}$ images, the accuracy of 3D models places within the scan resolution [28]. In order to use optimal parameter settings for segmentation procedure from CT scanning to virtual model reconstruction, in the present study, we used certified commercial software $^{1}$ that specifies accuracy is below scanning

\footnotetext{
${ }^{1}$ Mimics 22.0, Materialise NV, Belgium.
} 
resolution and clinical relevance [29]. This means the bone models can be used for presurgical rehearsal and further clinically relevant applications in bone surgery $[3,30]$.

A limitation of our study was its focus on mediumsized canine tibias (length:150-180 mm) because linear RMS errors become larger with bone model size [11]. The TPLO procedure requires extensive experience and is technically challenging in small and medium-sized dogs [31]. Therefore, our tibia model may serve as a patient-specific surgical model for TPLO in mediumsized dog breeds before and during surgery. However, geometric accuracy may vary for different tibial sizes. Another limitation of the study is that we did not evaluate the consistency/reproducibility (intraobserver repeatability) of our measurement methods. It is therefore not possible to know how much of the error in our measurements results from "noise" (i.e., the error of our measurement method). If this error were to be known, it would allow for better evaluation of the true impact of $\mathrm{H}_{2} \mathrm{O}_{2}$ sterilization. The small RMS error in $E_{\text {GAS-FDM }}$ suggests that our measurements were highly repeatable.

The CT-derived data were not compared with bone gross anatomy in this study. One study reported that the articular surface derived from CT images in small bones (e.g. feline radius) had dimensional errors and lacked precision when used to plan limb corrective osteotomy [32]. However, CT imaging can still be used to accurately evaluate and represent musculoskeletal anatomy [33-36] because the average deviation of CT-based human femur models from a bone-surface scan is negligible and clinically acceptable [23]. Therefore, further canine tibia cadaver reverse 3D scanning would be beneficial for ensuring geometric accuracy with respect to gross anatomy.

\section{Conclusion}

In veterinary medicine, the increasing demand for improved visualisation and surgical outcomes have made 3D-printed anatomical models and patient-specific guides popular, and they are becoming common for implant contouring, surgery rehearsal, client communication, and education. Our study demonstrated the high geometric accuracy of an FDM-printed ABS tibia model when compared with CT data. Furthermore, surface curvature deviation of the model after $\mathrm{H}_{2} \mathrm{O}_{2}$ plasma sterilisation was minimal. Therefore, preoperative plate contouring and surgical planning using our tibia model may reduce surgical time and achieve adequate bone reproduction. Additionally, our model may be used during operations because it has nearly the same surface curvature as that of real bone. Additional studies evaluating the geometric accuracy of patient-specific 3D-printed cutting guides for intraoperative use would be useful as they will help improve assessment of models used in a clinical setting.

\section{Methods}

\section{Sample collection}

All experimental protocols complied with the guidelines for the care and use of animals and were approved by the Institutional Animal Care and Use Committee of National Taiwan University (NTU108-EL-00015). Six mixed-breed skeletally mature canine cadavers (Mean body weight: $12 \mathrm{~kg}$ (10-15); 3 female and 3 male) were used and informed consent obtained from the animal shelter. The dogs' deaths were unrelated to this study. The dogs had no known histories of orthopaedic surgery. Coxofemoral amputation was performed on all 6 cadavers, and the 12 pelvic limbs were covered with saline-rinsed gauze and stored at $-20^{\circ} \mathrm{C}$. All experiments were performed $24 \mathrm{~h}$ after the pelvic limbs were thawed at room temperature.

\section{D-printed tibia model}

To obtain a 3D reconstruction, all the limbs underwent CT [1] imaging on a 128-slice CT scanner (Ingenuity Core 128, Philips Medical Systems, Cleveland, OH, USA). All limbs were placed in a caudal extension position with tibial diaphysis being perpendicular to the plane of imaging. Scanning was conducted at $120 \mathrm{kV}$ and $105-115 \mathrm{~mA} \mathrm{[10]}$. The scanning procedure was performed using $0.5 \mathrm{~mm}$ CT slices (resolution $512 \times 512$ pixels, pixels spacing $=0.43 \mathrm{~mm} /$ pixels) around the whole limb. Image preprocessing was performed following the procedure given by the commercial software ${ }^{1}$ : First, we used a threshold value at $220-3000$ HU to separate bone and soft tissue. Segmentation was performed to extract the tibia. The 3D tibial model was reconstructed using a high-frequency algorithm (Marching Cubes), and then smoothing performed. Finally, the 3D tibial model was exported as standard tessellation language (STL)-format files.

Both tibias of each dog were printed to be the same size as the cadavers' tibias. The tibia models were printed on a $3 \mathrm{D}$ printer. The printing material was $\mathrm{ABS}$ with a layer height of $0.2 \mathrm{~mm}$. Additive manufacturing error relates to layer height at $0.2 \mathrm{~mm}(\mathrm{Up} \mathrm{Box}+$, Milpitas, CA, USA). All tibia models were printed with the long-bone axis parallel to the printer surface and the proximal-medial side facing up with a substantial ABSprinted support structure underneath. After printing, the ABS base structure was gently removed manually from the tibia bone models. There was no further postprocessing performed on the tibial bone models.

Tibia morphological analysis before and after sterilisation After careful harvesting of the 12 tibia models, reverseengineering technology was used to analyse their errors [37]. The tibias were first scanned using an optical scanner (Breuckmann smartSCAN 3D, AICON 3D Systems, 
Braunschweig, Germany) with an accuracy of $0.02 \mathrm{~mm}$, and 3D virtual (point cloud) models (3D-FDM) were obtained from the images of the scanned 3D-printed tibia models. The scanned images were stacked and decomposed into multiple points to form the test model. The virtual model (3D-CT) was obtained from the original tibia CT images and decomposed into multiple points to form the reference model. Subsequently, the differences between the 3D-FDM and 3D-CT models were compared on a point-by-point basis by superimposing the two virtual models with software ${ }^{2}$, and calculations were performed using the closest projection point algorithm and Newton's method [38, 39]. A 2-phase superimposing process was performed: an initial manual labelling of bony structures on tibial bone models was followed by a second automated registration. Additionally, root mean square (RMS) errors were calculated. RMS errors are generally used to assess the mean value of errors and are typically used as a criterion for assessing the similarity of two $\mathrm{N}$-dimensional vector sets after optimal superimposition [40]. A higher RMS value indicates a larger error. The superimposition results were also illustrated by a colour difference map (Fig. 1a). The maximum and minimum values of the colour difference map were + 0.5000 and $-0.5000 \mathrm{~mm}$, respectively.

A low-temperature $\mathrm{H}_{2} \mathrm{O}_{2}$ STERRAD NX steriliser (50 $\min /$ cycle, temperature $<55^{\circ} \mathrm{C}$, Johnson \& Johnson Company) was used to sterilise the printed tibia models. The sterilised tibias were scanned a second time, and 3D virtual models (3D-GAS) were reconstructed. The superimposition results were also illustrated by a colour difference map (Fig. 1b). The maximum and minimum values of the colour difference map were +0.2000 and $0.2000 \mathrm{~mm}$, respectively. The difference between the 3DGAS and 3D-FDM was also evaluated using the closest projection point algorithm and Newton's method [38, 39]. Both the manufacturing process and sterilization can influence geometric accuracy in printed tibial models. We wanted to identify which of them impacted tibial models the most. Consequently, two comparisons were made: $\mathrm{E}_{\mathrm{FDM}-\mathrm{CT}}$ (3D-FDM vs. 3D-CT) and $\mathrm{E}_{\mathrm{GAS}-\mathrm{FDM}}$ (3D-GAS vs. 3D-FDM). Reverse-engineering software $^{2}$ was employed for error analysis among all models.

\section{Morphological analysis at the tibia cross-sectional profile} Following the previous mentioned superimposition of the two virtual models (Fig. 1), tibia length was defined as the longest distance from the proximal to distal end in the craniocaudal view of the $3 \mathrm{D}$ virtual model. The cross-sectional plane was simulated on the 3D virtual model at: $5,15,25,50,75,85$, and $95 \%$ of the previously

\footnotetext{
${ }^{2}$ RevCAD, PouYuen Tech Corp., Taoyuan, Taiwan.
}

recorded tibia length, and the contour points where the tangential plane intersected the model were extracted as point cloud data. A total of seven cross-sectional datasets were obtained for each tibia model and the RMS value was calculated through comparison of $\mathrm{E}_{\mathrm{FDM}-\mathrm{CT}}$ and $\mathrm{E}_{\text {GAS-FDM }}$ (Fig. 3, Table 2).

\section{Statistical analysis}

A Mann-Whitney $U$ test was conducted to evaluate the RMS mean differences between $\mathrm{E}_{\mathrm{FDM} \text {-CT }}$ and $\mathrm{E}_{\mathrm{GAS} \text {-FDM. }}$ A one-way analysis of variance was used to test the RMS mean differences among the seven cross-sectional datasets in each group. All statistical analyses were performed using GraphPad Prism 8.0 (GraphPad Software, Inc., San Diego, CA, USA). The significance level was set at $P<0.05$.

\section{Abbreviations \\ 3D: Three-dimensional; ABS: Acrylonitrile butadiene styrene; FDM: Fused deposition modelling; $\mathrm{H}_{2} \mathrm{O}_{2}$ : Hydrogen peroxide; RMS: Root mean square; TPLO: Tibial plateau levelling osteotomy; CT: Computed tomography; STL: Standard tessellation language}

\section{Acknowledgements}

The authors thank the staff in the National Applied Research Laboratories, Taiwan Instrument Research Institute for help in CT scanning and optical scans,

\section{Authors' contributions}

ILL conceived of and requested the research grant. ILL and CPH designed the study. ILL, CPH, and CHF performed the experiments. ILL, CPH, CSL, CHF, NYC, CWT, and CMC contributed in analyzing the results, writing, and reviewing the manuscript. The authors read and approved the manuscript.

\section{Funding}

This study was funded by a grant from National Taiwan University- Yonglin Humane Project (grant numbers FB004-6, 2018). The funding body had no role in the design of the study and collection, analysis, and interpretation of data nor in writing the manuscript.

\section{Availability of data and materials}

All data generated or analyzed during this study are included in this published article and available from the corresponding author on reasonable request.

\section{Ethics approval and consent to participate}

The study was approved by the Institutional Animal Care and Use Committee of National Taiwan University (NTU108-EL-00015) and conducted by trained professionals. The informed consent for the study was obtained from the animal shelter. No additional permissions were required for sample collection from canines.

Consent for publication

Not applicable. The manuscript does not contain any human data.

\section{Competing interests}

The authors declare that they have no competing interests.

\section{Author details}

'High Speed 3D Printing Research Center, National Taiwan University of Science and Technology, Taipei, Taiwan. ${ }^{2}$ Department and Graduate Institute of Veterinary Medicine, School of Veterinary Medicine, National Taiwan University, Taipei, Taiwan. Institute of Veterinary Clinical Science, School of Veterinary Medicine, National Taiwan University, Taipei, Taiwan. ${ }^{4} \mathrm{National}$ Applied Research Laboratories, Taiwan Instrument Research Institute, Hsinchu, Taiwan. 
Received: 17 June 2020 Accepted: 23 November 2020

Published online: 09 December 2020

\section{References}

1. Tack P, Victor J, Gemmel P, Annemans L. 3D-printing techniques in a medical setting: a systematic literature review. Biomed Eng Online. 2016; 15(1):115.

2. Kim GB, Lee S, Kim H, Yang DH, Kim YH, Kyung YS, et al. Three-dimensional printing: basic principles and applications in medicine and radiology. Korean J Radiol. 2016;17(2):182-97.

3. Hoang D, Perrault D, Stevanovic M, Ghiassi A. Surgical applications of threedimensional printing: a review of the current literature \& how to get started. Ann Transl Med. 2016:4(23):456.

4. DeTora MD, Boudrieau RJ. Complex angular and torsional deformities (distal femoral malunions). Preoperative planning using stereolithography and surgical correction with locking plate fixation in four dogs. Vet Comp Orthop Traumatol. 2016:29(5):416-25.

5. Hespel AM, Wilhite R, Hudson J. Invited review--applications for 3D printers in veterinary medicine. Vet Radiol Ultrasound. 2014;55(4):347-58

6. Harrysson OLA, Cormier DR, Marcellin-Little DJ, Jajal K. Rapid prototyping for treatment of canine limb deformities. Rapid Prototyp J. 2003;9(1):37-42.

7. Marcellin-Little DJ, Harrysson OLA, Cansizoglu O. In vitro evaluation of a custom cutting jig and custom plate for canine tibial plateau leveling. Am J Vet Res. 2008;69(7):961-6.

8. Winer JN, Verstraete FJM, Cissell DD, Lucero S, Athanasiou KA, Arzi B. The application of 3-dimensional printing for preoperative planning in oral and maxillofacial surgery in dogs and cats. Vet Surg. 2017;46(7):942-51.

9. Oxley B. Bilateral shoulder arthrodesis in a Pekinese using three-dimensional printed patient-specific osteotomy and reduction guides. Vet Comp Orthop Traumatol. 2017:30(3):230-6.

10. Fitzwater KL, Marcellin-Little DJ, Harrysson OL, Osborne JA, Poindexter EC. Evaluation of the effect of computed tomography scan protocols and freeform fabrication methods on bone biomodel accuracy. Am J Vet Res. 2011;72(9):1178-85.

11. Cone JA, Martin TM, Marcellin-Little DJ, Harrysson OLA, Griffith EH. Accuracy and repeatability of long-bone replicas of small animals fabricated by use of low-end and high-end commercial three-dimensional printers. Am J Vet Res. 2017;78(8):900-5.

12. Boursier JF, Fournet A, Bassanino J, Manassero M, Bedu AS, Leperlier D. Reproducibility, accuracy and effect of autoclave sterilization on a thermoplastic three-dimensional model printed by a desktop fused deposition modelling three-dimensional printer. Vet Comp Orthop Traumatol. 2018;31(6):422-30.

13. Columbus L. The State of 3D Printing [Internet]. 2018 [cited 2020 Aug 21]. Available from: https://www.forbes.com/sites/louiscolumbus/2018/05/30/ the-state-of-3d-printing-2018/.

14. Katatny IE, Masood SH, Morsi YS. Evaluation and validation of the shape accuracy of FDM fabricated medical models. Adv Mat Res. 2009:83-86:275-80.

15. Petropolis C, Kozan D, Sigurdson L. Accuracy of medical models made by consumer-grade fused deposition modelling printers. Plast Surg (Oakv). 2015;23(2):91-4

16. Wei YP, Lai YC, Chang WN. Anatomic three-dimensional model-assisted surgical planning for treatment of pediatric hip dislocation due to osteomyelitis. J Int Med Res. 2020:48(2):300060519854288.

17. Hall EL, Baines S, Bilmont A, Oxley B. Accuracy of patient-specific threedimensional-printed osteotomy and reduction guides for distal femoral osteotomy in dogs with medial patella luxation. Vet Surg. 2019;48(4):584-91.

18. Rozema FR, Bos RR, Boering G, van Asten JA, Nijenhuis AJ, Pennings AJ. The effects of different steam-sterilization programs on material properties of poly(L-lactide). J Appl Biomater. 1991;2(1):23-8.

19. Shaheen E, Alhelwani A, Van De Casteele E, Politis C, Jacobs R. Evaluation of dimensional changes of 3D printed models after sterilization: a pilot study. Open Dent J. 2018;12:72-9.

20. Kuczko W, Wichniarek R, Górski F, Banaszewski J. Influence of sterilization of a product manufactured using FDM technology on its dimensional accuracy. Adv Sci Technol Res J. 2018;12(1):74-9.

21. Sosnowski EP, Morrison J. Sterilization of medical 3d printed plastics: Is H O vapour suitable? In:Proceeding of the 40 Canadian Medical and Biological Engineering Conference, 2017. Winnipeg: The Canadian Medical and Biological Engineering Society; 2017.
22. McCreanor V, Graves N. An economic analysis of the benefits of sterilizing medical instruments in low-temperature systems instead of steam. Am J Infect Control. 2017;45(7):756-60.

23. Soodmand E, Kluess D, Varady PA, Cichon R, Schwarze M, Gehweiler D, et al. Interlaboratory comparison of femur surface reconstruction from $\mathrm{CT}$ data compared to reference optical 3D scan. Biomed Eng Online. 2018;17(1):29.

24. Pinto JM, Arrieta C, Andia ME, Uribe S, Ramos-Grez J, Vargas A, et al. Sensitivity analysis of geometric errors in additive manufacturing medical models. Med Eng Phys. 2015;37(3):328-34

25. Alharbi N, Osman RB, Wismeijer D. Factors influencing the dimensional accuracy of 3D-printed full-coverage dental restorations using stereolithography technology. Int J Prosthodont. 2016;29(5):503-10.

26. Dey A, Yodo N. A systematic survey of FDM process parameter optimization and their influence on part characteristics. J Manuf Mater Process. 2019;3(3): 64.

27. Rathnayaka K, Sahama T, Schuetz MA, Schmutz B. Effects of CT image segmentation methods on the accuracy of long bone 3D reconstructions. Med Eng Phys. 2011;33(2):226-33.

28. Noser H, Heldstab T, Schmutz B, Kamer L. Typical accuracy and quality control of a process for creating CT-based virtual bone models. J Digit Imaging. 2011;24(3):437-45.

29. Gelaude F, Vander Sloten J, Lauwers B. Accuracy assessment of CT-based outer surface femur meshes. Comput Aided Surg. 2008;13(4):188-99.

30. Oth O, Dauchot C, Orellana M, Glineur R. How to sterilize 3D printed objects for surgical use? An evaluation of the volumetric deformation of 3D-printed genioplasty guide in PLA and PETG after sterilization by low-temperature hydrogen peroxide gas plasma. Open Dent J. 2019;13(1):410-7.

31. Garnett SD, Daye RM. Short-term complications associated with TPLO in dogs using 2.0 and 2.7 mm plates. J Am Anim Hosp Assoc. 2014;50(6):396-404.

32. Webster CE, Marcellin-Little DJ, Koballa EM, Stallrich JW, Harrysson OLA. Evaluation of the geometric accuracy of computed tomography and microcomputed tomography of the articular surface of the distal portion of the radius of cats. Am J Vet Res. 2019:80(10):976-84.

33. Jurik AG, Albrechtsen J. The use of computed tomography with two- and three-dimensional reconstructions in the diagnosis of three- and four-part fractures of the proximal humerus. Clin Radiol. 1994;49(11):800-4.

34. Kuszyk BS, Heath DG, Bliss DF, Fishman EK. Skeletal 3-D CT: advantages of volume rendering over surface rendering. Skeletal Radiol. 1996;25(3):207-14.

35. Ney DR, Fishman EK, Kawashima A, Robertson DD Jr, Scott WW. Comparison of helical and serial CT with regard to three-dimensional imaging of musculoskeletal anatomy. Radiology. 1992;185(3):865-9.

36. Larguier L, Hespel AM, Jamet N, Mercier E, Jouan D, Jardel N, et al. Accuracy and precision of measurements performed on three-dimensional printed pelvises when compared to computed tomography measurements. J Vet Sci. 2019;20(3):e22

37. Leng S, McGee K, Morris J, Alexander A, Kuhlmann J, Vrieze T, et al. Anatomic modeling using 3D printing: quality assurance and optimization. 3D Print Med. 2017:3(1):6.

38. Arun KS, Huang TS, Blostein SD. Least-squares fitting of two 3-d point sets IEEE Trans Pattern Anal Mach Intell. 1987:9(5):698-700.

39. Yau H-T, Chen C-Y, Wilhelm RG. Registration and integration of multiple laser scanned data for reverse engineering of complex 3D models. Int J Prod Res. 2010;38(2):269-85.

40. Jeong YG, Lee WS, Lee KB. Accuracy evaluation of dental models manufactured by CAD/CAM milling method and 3D printing method. J Adv Prosthodont. 2018;10(3):245-51.

\section{Publisher's Note}

Springer Nature remains neutral with regard to jurisdictional claims in published maps and institutional affiliations. 